\title{
Behavior of a 2-Dimensional Ceiling Jet Flow: A Beamed Ceiling Configuration
}

\author{
CARL C. KOSLOWSKI ${ }^{1}$ and VAHID MOTEVALLI \\ Center for Firesafety Studies \\ Worcester Polytechnic institute \\ Worcester, MA 01609
}

\begin{abstract}
Detailed measurements of velocity and temperature in a primary channel of a beamed ceiling are presented in this paper as well as flow spillage under the beams. The data has been used to validate the empirical relation developed by Delichatsios and modify it for use in a more general situation. The primary focus has been to characterize the flow for beam depths that result in a ceiling jet to be in the transition between smooth ceiling jet flow and a corridor flow. As a result of this study, a modified empirical relation to calculate the ceiling jet temperature in a primary channel based on the ceiling heat transfer and the beam to ceiling height ratio has been presented here. The results tend to show that primary channel flow approaches a corridor flow for beam depth higher than $15 \%$ to $20 \%$ of the fire to ceiling height.
\end{abstract}

KEYWORDS: Ceiling jet velocity and temperature, Heat detection, turbulent flow, beams.

\section{INTRODUCTION}

Ceilings may have obstructions such as beams, or surface mounted light fixtures, protruding as little as $10 \mathrm{~cm}$ ( 4 inches) below the ceiling. In case of beams, variations in the beam span, depth, width, all can affect the ceiling jet characteristics. In each of these cases, the ceiling obstruction affects the ceiling jet characteristics and can alter heat detector, automatic sprinkler and smoke detector response. This paper addresses those beamed ceiling configurations where:

1. The ceiling obstructions create channels which does not restrict the flow of the ceiling jet in the direction parallel to the ceiling obstructions.

2. The beam half-span, $l_{\mathrm{b}}$, is greater than the plume impingement region, $l_{\mathrm{b}}>0.2 \mathrm{H}$, where $\mathrm{H}$ is the ceiling height above the fire source.

In this paper, two key regions of the ceiling with beams need to be defined. First,

Currently with Fire Protection Design, Inc, Warren, MA. 
the primary bay is defined as an unrestricted channel wherein the fire plume impinges, and second, the secondary bay, which is an unrestricted channel adjoining the primary channel, at either side, where the fire induced flow is reached by spillage under the primary bay beams (see Figures $1 \mathrm{a} \& \mathrm{~b}$ ).

\section{Smooth vs. Beamed Ceiling Jet Flow}

Extensive studies have been conducted which provide characterization of the smooth unconfined ceiling jet. Research by Alpert [1], Heskestad and Delichatsios [2], and Motevalli and Marks [3] have provided theory and correlations which allow characterization of the fire induced ceiling jet under a smooth unconfined ceiling. Additional research has

been conducted by many other investigators, however, the results of these studies are

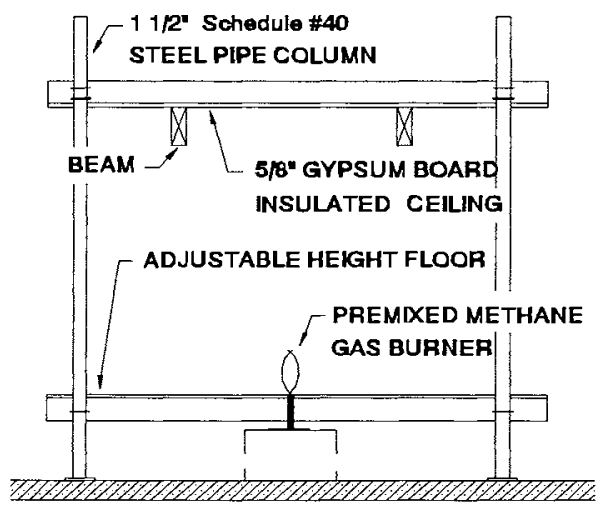
sufficient for the intent of this discussion.

Figure 1a - Schematic of the apparatus In each of these studies, correlations have been developed which offer a functional relationship between the radial position, $r$, the ceiling height above the fire, $H$, the fire size, $\dot{Q}$, and the temperature and velocity of the ceiling jet.

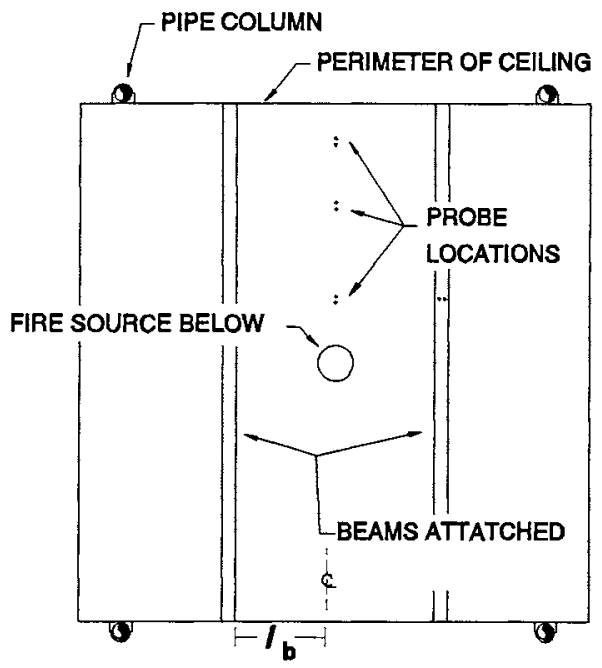

Figure $\mathbf{l b}$ - Top view - Measurement locations

Under beamed ceilings, the flow cannot immediately be classified as axisymmetric or two-dimensional without a concern for the beam depth and spacing. As the beam depth increases, the ceiling jet flow is transformed into a twodimensional, corridor-type, flow. However, between the smooth unconfined ceiling and corridor flow lies the partially confined ceiling jet. Under this condition, the ceiling jet will exhibit characteristics associated with both the smooth unconfined ceiling, and the corridor flow. As a result, the parameters discussed for the smooth unconfined ceiling, (i.e., $\mathrm{r} / \mathrm{H}$ and $\mathrm{Q}^{*}$ ), will also be important in the analysis of beamed ceiling jet flow.

Heskestad [4] using reduced scale of 1:12.5 for beamed ceilings, pointed out that sprinkler actuation may be enhanced due to the channeling of the flow if a sprinkler was located in each ceiling bay. He also identified the key parameters affecting the beamed ceiling flow. Bill et al. [5] examined the effect of beamed and cathedral ceilings on residential sprinkler performance. They recognized the inability to predict sprinkler response as a result of numerous possible 
configurations making correlation of the data quite difficult.

In a more recent work, Koslowski and Motevalli [6] conducted an extensive study of the ceiling jet flow for a beamed ceiling using small-scale apparatus. Their study included measurement of velocity and temperature beyond a beam as well as within the primary channel. The results concentrating on the ceiling jet characteristics beyond a beam has been published previously [6]. This paper focuses on the flow behavior within a channel created by beams where the fire plume impinges and for the intermediate range of beam depths (i.e. $0<\mathrm{d} / \mathrm{H}<0.2$ ). The amount of flow spillage under the beam and flow characteristics for such a $\mathrm{d} / \mathrm{H}$ range are of interest for sensor placement under the obstructed ceilings.

\section{THEORY AND BACKGROUND}

Delichatsios [7] has presented the bulk of the theory regarding flows within a primary channel. His paper also presents correlations for the temperature and velocity of the ceiling jet within this channel. According to his work, the ceiling jet is initially axi-symmetric until it is intercepted by the beams. At this point a transition region begins until the flow converges to a two dimensional, channel flow. Upon intersection of the ceiling jet with the beams, a portion of the gases escape under the beam and into adjacent channels. The remainder of the gases flow within the channel in opposite directions. However, if the beams are sufficiently deep, large $\mathrm{d} / \mathrm{H}$, the leakage under the beams becomes small, and all of the fire gases are restricted to the primary channel. According to Delichatsios, this occurs at a beam depth to ceiling height ratio, $\mathrm{d} / \mathrm{H}$, of 0.2 . Our work [6] has essentially confirmed this by showing that a $\mathrm{d} / \mathrm{H} \approx 0.15$ produces an $\approx 80 \%$ reduction in flows under the beam at the closet radial location from the plume centerline. An asymptotic reduction toward a corridor flow occurs for $\mathrm{d} / \mathrm{H}>0.15$.

\section{The Obstructed Ceiling Jet - Primary Bay}

Within the primary channel, the flow is axi-symmetric until the ceiling jet flow approaches the beam. The presence of the beams do not affect the ceiling jet flow within the plume impingement area if $\mathrm{l}_{\mathrm{b}} / \mathrm{H}>0.2$. Upon reaching a distance from the fire source equal to the beam spacing, $Y=l_{b}$, the ceiling jet transitions to a two-dimensional flow pattern, parallel to the span of the beam. According to Delichatsios [7], two possibilities for the primary channel flow develops upon intersection with the beam.

1. The flow is a turbulent one dimensional jet with high Froude number (Fr). In this case, air entrainment is significant.

2. A subcritical $(\mathrm{Fr}<1.0)$ flow, as the result of a hydraulic jump, which occurs as the turning plume encounters the beams, with a transition to a critical flow ( $F r=1)$ due to transformation to a 2-D flow (Delichatsios assumed a 1-D flow) between the beams.

The latter case is postulated to occur in accordance with the results of Delichatsios [7]. This flow regime contains no air entrainment into the ceiling jet resulting in limited reductions in both temperature and velocity. In addition, the ceiling jet flow is no longer expanding axi-symmetrically, resulting in smaller reductions in the ceiling jet temperature and velocity. Since little air entrainment is expected, Delichatsios states that reductions in 
the temperature of the ceiling jet are solely due to heat transfer to the ceiling.

Delichatsios [7] showed that the temperature of the fire plume at ceiling impingement, for the case of corridor flow, can be described by:

$$
\frac{\Delta T}{\Delta T_{0}}=0.281\left(\frac{H}{l_{b}}\right)^{\frac{1}{3}}
$$

He arrived at equation (1) based on the conservation equations of momentum and energy. This shows an inverse dependency of $\Delta \mathrm{T}$ on $l_{b} / \mathrm{H}$ alone.

For the flow beyond the turning region, Delichatsios [7] developed the following relation for an average temperature within the primary channel.

$$
\frac{\Delta T}{\Delta T_{0}}\left(\frac{l_{b}}{H}\right)^{\frac{1}{3}}=a \exp \left[-6.67 S t\left(\frac{Y}{H}\right)\left(\frac{l_{b}}{H}\right)^{\frac{1}{3}}\right]
$$

where: $\quad \mathrm{Y}=$ distance along channel (or) corridor

$\Delta \mathrm{T}_{0}=$ temperature rise at the ceiling center line plume temperature.

$\mathrm{St}=\mathrm{h}_{\mathrm{c}} / \rho \mathrm{U} \mathrm{c}_{\mathrm{p}}$

$\mathrm{a}=$ pre-exponential factor, based on ceiling properties and configuration.

and the correlation of Eq. (2) is restricted to the following range of parameters:

$$
\mathrm{d} / \mathrm{H} \geq 0.20 ; \quad \mathrm{Y}>1_{\mathrm{b}} ; \quad 0.5<(\mathrm{Y} / \mathrm{H})\left(1_{\mathrm{b}} / \mathrm{H}\right)^{1 / 3}<3.0 ; \quad \mathrm{b}_{\mathrm{b}}>0.15 \mathrm{H}
$$

Equation (2), was developed based on assumptions of; no reduction in ceiling jet velocity, no air entrainment, constant gas properties, a constant ceiling temperature, and that the heat transfer coefficient is proportional to the flow velocity. Delichatsios reports the value of the Stanton number, St, to be 0.03 . The constant Stanton number indicates that the combined effect of variations in the heat transfer coefficient, ceiling jet velocity and flow density results in a constant, which supports the assumption of limited air entrainment. The only configurations tested experimentally by Heskestad and Delichatsios [8] were for $d / H$ equal to 0.111 and 0.20 . Therefore, the results of their study are only applicable to those ceiling configurations. The value of the pre-exponential factor, "a", has been reported by Delichatsios for a beam depths of 0.20 and 0.111 to be 0.29 and 0.24 , respectively.

Although temperature and velocity profile data were not collected by Delichatsios, he states that the ceiling jet profiles for $Y>l_{b}$ should be uniform. Temperature and velocity profile data have been measured in this work to investigate this statement.

Based on Delichatsios postulation [7], the following would be expected for a subcritical flow within the primary bay:

1. Temperature and velocity profiles which are non-Gaussian and flat.

2. Limited air entrainment.

3. Temperature reductions as a function of $\mathrm{Y} / \mathrm{H}$ which are a result of heat transfer to the ceiling.

An inherent difficulty with Eq. (2) is the relation between the center line plume temperature, $\Delta \mathrm{T}_{0}$, and the fire size. Heskestad and Delichatsios [8] utilized a value of $\Delta \mathrm{T}_{0} /\left(\mathrm{Q}^{2 / 3} \mathrm{Z}^{-5 / 3}\right)=25.5$, however, it has been shown that this value can vary significantly depending on the fuel source, geometry, environmental conditions and measurement approach [9]. A non-dimensional form of Eq. (2) as a function of $\Delta T^{*}$ is presented (see Eq. 
3) to reduce the aforementioned uncertainties. Notice also that the constant "a" is replaced with a constant " $b$ " in the following equation:

$$
\Delta T^{*}=b\left(\frac{H}{l_{b}}\right)^{\frac{1}{3}} \exp \left[-6.67 \text { St }\left(\frac{Y}{H}\right)\left(\frac{l_{b}}{H}\right)^{\frac{1}{3}}\right]
$$

where: $\quad b=$ pre-exponential factor, accounting for $\mathrm{d} / \mathrm{H}$; and,

$$
\Delta T^{*}=\frac{\Delta T}{Q^{* 2 / 3} T_{\infty}} \quad \text { where: } \quad Q^{*}=\frac{\left(1-\lambda_{p}\right) Q}{\rho c_{p} T_{\infty} g^{1 / 2} H^{5 / 2}}
$$

In Eq. (4), $\lambda_{\mathrm{T}}$ is the fire source radiant fraction loss.

This paper provides detailed measurements to investigate the validity of Eq. (3) by examining the temperature in the primary channel as a function of beam depth. By varying $\mathrm{d} / \mathrm{H}$, the dependency of $\mathbf{b}$ as a function of beam depth will be investigated.

It is useful to point out two important boundary conditions which are expected to be maintained throughout this analysis. At $\mathrm{d} / \mathrm{H}=0$, the beamed ceiling is actually a smooth unconfined ceiling. At $\mathrm{d} / \mathrm{H}=1.0$, the configuration's upper limit (i.e. a corridor) is reached.

\section{EXPERIMENTS}

Extensive experiments, including flow visualization and measurement of ceiling jet temperature and velocity were conducted. The apparatus used for simulating the unconfined beamed ceiling configuration consisted of an insulated gypsum board ceiling, a premixed methane burner, a Cross Correlation Velocimetry (CCV) probe and data acquisition system. Figure 1a shows the schematic of the apparatus. Detailed description of the apparatus has been provided by Koslowski and Motevalli [6] elsewhere.

\section{Ceiling Jet Measurements}

Ceiling jet temperature measurements were obtained with a thermocouple probe consisting of $18 \mathrm{E}$-Type (chromel-constantan) thermocouples with a wire diameter of $0.025 \mathrm{~mm}$ $(0.001 ")$. During the experiments, the probe was positioned under the beam and at several locations away from the plume turning region, along the centerline of the primary bay.

Cross Correlation Velocimetry (CCV) was utilized to measure the velocity and temperature of the ceiling jet simultaneously. This method has been extensively discussed and validated by Motevalli et al. [10]. Six thermocouple pairs, separated by a known distance (typically $2-3 \mathrm{~cm}$ ) were used to obtain velocity data at several points beneath the ceiling. Each thermocouple pair was used to obtain a single point measurement of the ceiling jet velocity and temperature. An additional 6 thermocouples were used to provide more details for the temperature profile. (see Koslowski [11] for details). 


\section{Experimental Range}

Table 1 contains a summary of the important variables examined in each experiment. The intent of the experiments was to choose a large range of $\mathrm{d} / \mathrm{H}$, and examine the effect of beam spacing, $l_{\mathrm{b}} / \mathrm{H}$. It has already been shown [6] that varying $Q^{*}$ did not influence the functional relationship temperature and velocity to $\mathrm{d} / \mathrm{H}$. Temperature data have also been collected during the ceiling heat-up (transient) period for each of the experiments [11]. The flow spillage under the beam and its characteristics, quantified by velocity and temperature profiles, were also examined. The details of the experiments have been reported Table 1 - Summary of experimental parameters

$\underline{Q}=0.0267, Q=5.2 \mathrm{~kW}, \underline{H=0.5 \mathrm{~m}}$

\begin{tabular}{|c|c|c|c|}
\hline Exp. \# & $\underline{\mathrm{d} / \mathrm{H}}$ & $\underline{l}_{b} \underline{\underline{H}}$ & $\underline{\mathbf{Y} / \mathbf{H}}$ \\
\hline 21 & 0.074 & 0.3 & $0.5,1,1.6$ \\
\hline 22 & 0.074 & 0.6 & $0.5,1$ \\
\hline 23 & 0.123 & 0.3 & $0.5,1,1.6$ \\
\hline 24 & 0.123 & 0.6 & $0.5,1$ \\
\hline 25 & 0.175 & 0.3 & $0.5,1,1.6$ \\
\hline 26 & 0.175 & 0.6 & $0.5,1$ \\
\hline 27 & 0.276 & 0.3 & $0.5,1,1.6$ \\
\hline 28 & 0.276 & 0.6 & $0.5,1$ \\
\hline
\end{tabular}

previously by Koslowski [11] and only

a sample of the results are presented here.

\section{RESULTS}

The temperature of the ceiling jet flowing under the beam (see Fig. 1b, point A), as a function of distance below the ceiling, is plotted in Figure 2 for various normalized beam depth $(d / H)$. The profiles have been non-dimensionalized according to Eq. (4) and then normalized with respect to the non-dimensional maximum smooth ceiling jet temperature $\left(\Delta \mathrm{T}_{\max , \mathrm{s}}^{*}\right)[3]$ at the equivalent radial location (i.e., $\mathrm{r} / \mathrm{H}=\mathrm{l}_{\mathrm{b}}$ ). The ceiling jet thickness is normalized by the thermal Gaussian width, $l_{\mathrm{T}}$, of the smooth ceiling jet [3] at the same equivalent radial location. The smooth ceiling temperature profile, based on Motevalli and Marks' correlation [3], is also displayed for comparison. The beam position is indicated by the cross hatched box for each beamed ceiling case.

For this case, the maximum temperature occurs at between $4-5 \mathrm{~cm}$ below the beam, or at approximately $\mathrm{z} / \mathrm{H}=0.049$ below the tip of the obstruction. According to Motevalli's correlations, the corresponding smooth ceiling maximum temperature occurs at about $4 \mathrm{~mm}$ below the ceiling, namely an increase in the ceiling jet thermal boundary layer of a factor of 10 [3]. The downward flow of the ceiling jet under the obstruction, producing a recirculation before and under the beam, causes this widening of the jet thickness and thus maximum temperature to occur at a much greater distance below the ceiling. The maximum temperature can be readily seen to decrease as the beam depth to height ratio increases.

The ceiling jet temperature profile is remarkably similar to that of the smooth ceiling jet, but much wider. The maximum temperature under the beam appears to occur within the recirculating zone under the beam. As in the case of the smooth ceiling temperature profile, a rapid decrease in temperature occurs below the beam once the maximum temperature is reached. From the figures and additional results (see [11]), it appears that beam spacing, $l_{b} / H$, may significantly affect the flow behavior under the obstruction, however, variation of $Q^{*}$ has a negligible effect on the ceiling jet maximum temperature compared to the smooth 
ceiling case. The independence of the normalized jet from $Q^{*}$ was also observed for the secondary bay flow [6]. Figure 2 clearly shows that as the beam depth increases, the spillage under the beam decreases and the maximum temperature position is quite close to the beam surface. A limited flow visualization, conducted previously [11], provides qualitative observations, which are in agreement with these results.

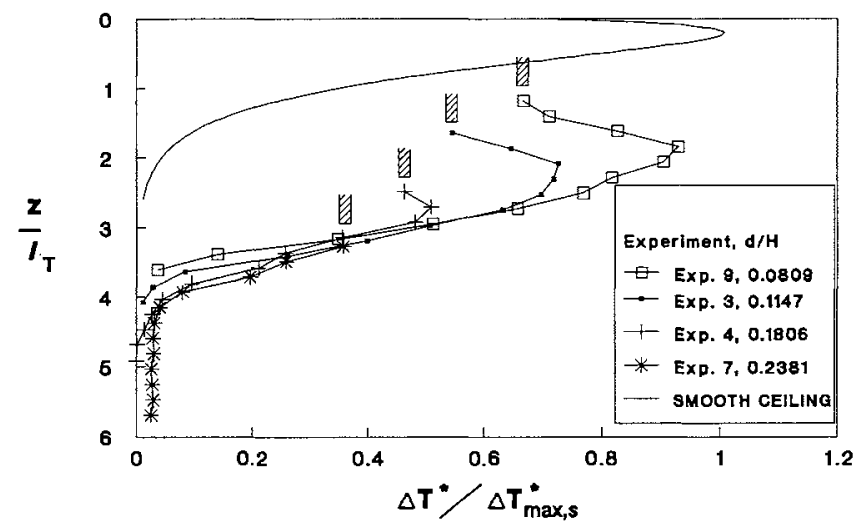

Figure 2 - Normalized and Non-dimensional temperature profiles under the beam for increasing $\mathrm{d} / \mathrm{H} . \mathrm{Q}^{*}=0.018$ and $l_{\mathrm{b}} / \mathrm{H}=0.5$.

\section{Temperature Profiles Within the Primary Bay}

Temperature profile data for experiments $21,23,25$, and 27 are displayed in Figures 3, 4, and 5. Each figure represents the temperature profiles at $\mathrm{Y} / \mathrm{H}$, locations of 0.5 , 1.0 a n 1.6 , respectively. The temperature profiles have been nondimensionalized and normalized the same way as for Figure 2 (note that, $\mathrm{r} / \mathrm{H}=\mathrm{Y} / \mathrm{H}$ ).

The ceiling jet maximum temperature, at $\mathrm{Y} / \mathrm{H}=0.5$, is reduced when compared to the smooth ceiling

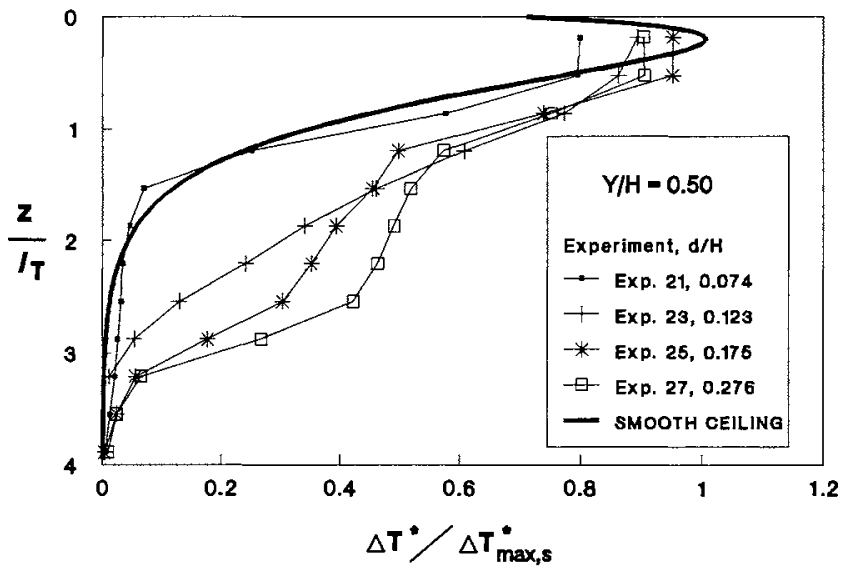

Figure 3 - Non-dimensional and normalized temperature profiles at the primary bay centerline. $Q^{*}=0.0267, l_{b} / H=0.3$. temperature. This can be attributed to increased entrainment and partial containment of the flow within the bay close to the impingement zone. As the beam depth is increased, the ceiling jet temperature is increased. This is simply a result of increased ceiling jet flow confinement caused by the beam.

Temperature profiles within the primary bay are not Gaussian and the profiles do not 
seem to be as "uniform" as predicted by Delichatsios. As $\mathrm{Y} / \mathrm{H}$ is increased, the profile become even less Gaussian, as seen in Figures 4 and 5 . For large $d / H$ conditions and a developed channel flow (Fig. 5), the temperature profiles approach a wave front profile shape. This seems to be consistent with the assumption of $\mathrm{Fr}<1$ flow stated by Delichatsios.

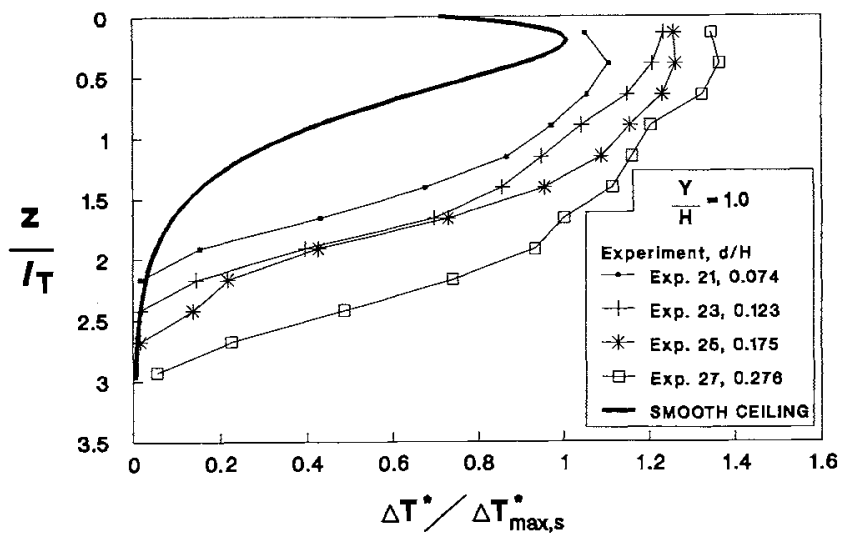

Figure 4 - Same as Figure 3, except at $\mathrm{Y} / \mathrm{H}=1.0$.

I $\mathrm{n}$ t h e s e Figures, the ceiling jet temperature profiles follow an analogous dependency on $\mathrm{d} / \mathrm{H}$. As the beam depth is increased, the ceiling jet temperature is increased. The maximum beam depth tested experimentally was $\mathrm{d} / \mathrm{H}=0.27$. At this point, the nondimensional maximum temperature $\left(\Delta \mathrm{T}^{*} / \Delta \mathrm{T}_{\text {max, }}^{*}\right)$ is 1.35 times the corresponding smooth ceiling value

for $\mathrm{Y} / \mathrm{H}=1.0$ as seen in Figure 5 and even higher $(\approx 1.5)$ for $\mathrm{Y} / \mathrm{H}=1.6$.

Although it is not evident in Figures $3-5$, the overall temperature of the ceiling jet reduces as the distance from the fire source is increased due to heat losses to the ceiling and beams. However, it can be seen that the nondimensional maximum temperature at $\mathrm{Y} / \mathrm{H}=1.6$ is larger $\mathrm{t} h$ a $\mathrm{n} h \mathrm{e}$ nondimensional maximum temperature at $\mathrm{Y} / \mathrm{H}=1.0$ for any ceiling configuration. From this information, it is clear that the temperature reduction of the obstructed

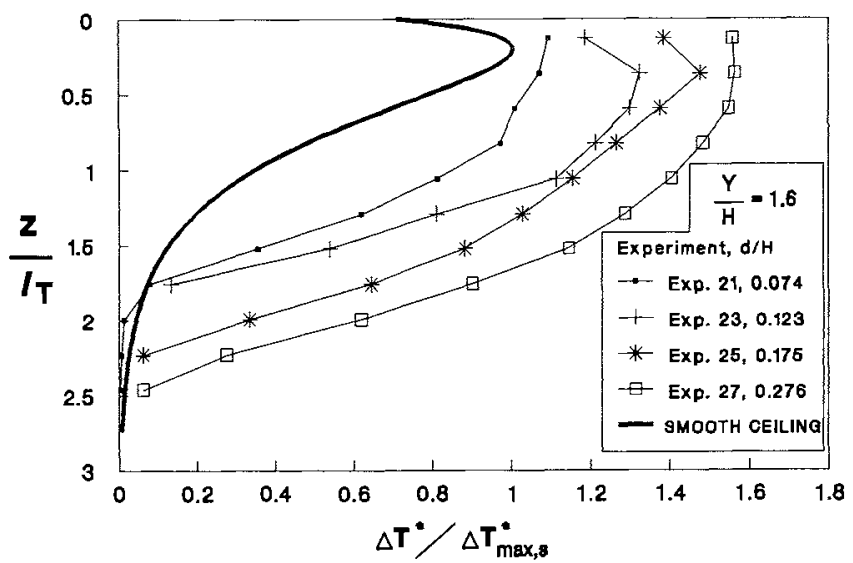

Figure 5 - Same as Figure 4 for $\mathrm{Y} / \mathrm{H}=1.6$, farthest from the impingement point ceiling jet is not as much as that which occurs for the smooth ceiling. This provides support for the assumptions of reduced air entrainment, as a result of sub-critical flow, and heat losses which occur only as a result of heat transfer to the ceiling.

The temperature profiles in Figure 3 indicate that at large $d / H$, experiments 25 and 27 , there is a sudden change in the slope of the curves. It appears that the inflection in the 
temperature profile occurs at approximately $\mathrm{z} / l_{\mathrm{T}}=1.3$. The profile shows a shooting boundary layer and a free jet region which is generated due to the flow containment by the beams and transition to a 2-D flow from an axi-symmetric condition. This behavior, interestingly, is seen for $d / H>0.15$. This is in total agreement with the critical $d / H$ value of about 0.15 determined for the secondary bay flow [6]. A close examination of Figures $3-5$ for $\mathrm{d} / \mathrm{H}>0.25$ indicates a widening of the ceiling jet layer and dissipation of the lower free jet flow. This seems to indicate that a hydraulic jump may have caused the initial twotier profile (Fig. 3, $\mathrm{d} / \mathrm{H}>0.15$ ) which is smoothed over as the flow proceeds down stream $(\mathrm{Y} / \mathrm{H}=1.0$ and 1.6$)$.

\section{Velocity Profile Data}

The ceiling jet velocity was measured within the primary channel for ceiling steady state conditions. Velocity profiles at $\mathrm{Y} / \mathrm{H}=1.0$ are shown in Figure 6. The velocity data contains more scatter (due to d ifficult y in measurements - the uniform flow within the primary channel reduces thermal fluctuations needed for the CCV technique). However, it is clear

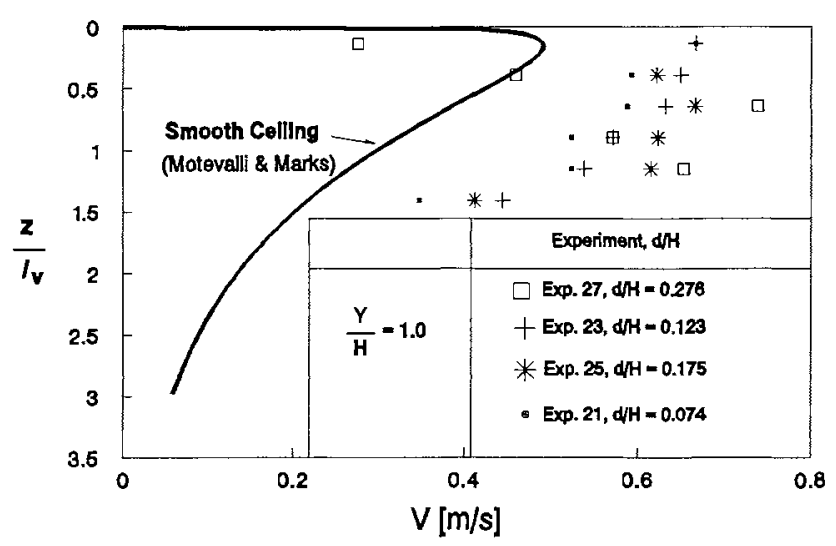

Figure 6 - Velocity profiles within the primary bay. $Q^{*}=0.0267$, $l_{\mathrm{b}} / \mathrm{H}=0.3$.

that magnitude of

velocity has increased significantly when compared to the smooth ceiling jet. The data indicates that the ceiling jet velocity profile is flatter than the smooth ceiling jet profile at the same radial location.

\section{Temperature Predictions in the Primary Bay Based on Heat Transfer Calculations}

The prediction of the temperature within the primary bay based on Delichatsios's relation (Eq. 3) is compared with the experimental results. Figure 7 presents the nondimensional temperature for corridor flow $(\mathrm{d} / \mathrm{H}=0.2)$ and for $\mathrm{d} / \mathrm{H}=0.111$ based on a pre-exponential factor and Stanton number determined experimentally by Delichatsios. The experimental data are from this study for the average nondimensional temperature from experiments $21,23,25$, and 27.

The trend of the experimental data appears to follow the theoretical prediction very well. However, the magnitude of the temperature predictions by Delichatsios [7] (which are based on measurements from [4]) are lower. Even for the smallest of beam depths, the ceiling jet temperature was recorded higher than the predicted value. This can be directly attributed to the more detailed temperature data collected and an improved measurement of 
the heat release rate, as well as the uncertainty in the Stanton number and pre-exponential factor $b$. The measurements by Heskestad and Delichatsios [4] are based on a single point measurement at a fixed distance below the ceiling while our averaged data are based on a detailed profile. In our work, twelve experimental data points were used to determine the temperature profile.

As noted, the Stanton number utilized to predict the temperature in Figure 7 was that obtained experimentally by Delichatsios [7]. However, differences in the ceiling construction alter this value. Therefore, values for both the pre-exponential factor, $b$, and the Stanton number, St, have been calculated based on the experimental results of this study. It should be noted that our calculations are bound by the measurement range of $0.5 \checkmark \mathrm{Y} / \mathrm{H} \checkmark 1.6$.

\section{Calculation of St and $b$}

\section{Calculation of}

both the preexponential factor and the Stanton number can be easily obtained based on multiple measured values. Simultaneous solutions of Eq. (3) at various positions results in a Stanton number of 0.04 (compared to 0.03 re ported by Delichatsios) and a preexponential factor, $b$, as a function of $\mathrm{d} / \mathrm{H}$ which is shown in

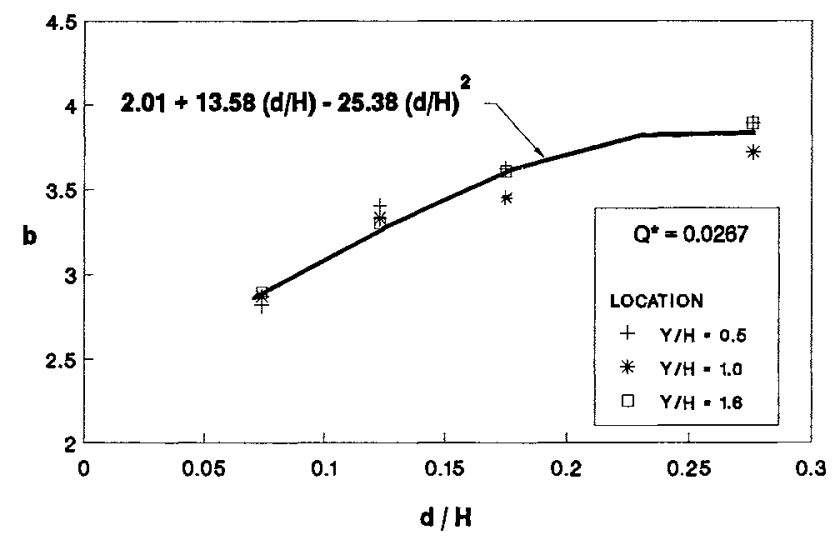

Figure 8 - Dependance of $\mathbf{b}$ on $\mathrm{d} / \mathrm{H}, l_{\mathrm{b}} / \mathrm{H}=0.3$.

Figure 8 . The trend of

this figure indicates strong dependency of $\mathbf{b}$ on $d / H$. Measurements of velocity and temperature within the secondary bay, has indicated that even for $\mathrm{d} / \mathrm{H}$ between 0.04 and 0.15 , there is a significant reduction in the velocity and temperature compared to the smooth unconfined conditions [6]. In this case, the largest increase in the exponential factor occurs near a $\mathrm{d} / \mathrm{H}=0.15$. Therefore, as the greatest reductions in the temperature occur in the 
secondary bay, the largest increases in the primary bay temperature occurs (i.e. the spillage to the secondary bay reduces greatly). When $\mathrm{d} / \mathrm{H}$ is greater than 0.15 , the temperature in both the primary and secondary bay is much less affected by increases in the beam depth.

Based on the analysis performed here and presented in Figure 8, the following correlation for evaluation of the pre-exponential factor (b) is developed.

$$
b=-25.38\left(\frac{d}{H}\right)^{2}+13.58\left(\frac{d}{H}\right)+2.01 \quad 0.5 \leq \frac{Y}{H} \leq 1.6
$$

Based on the determined functionality of the pre-exponential factor, Figure 8, and the Stanton number, St $=0.04$, Figure 9 shows the predicted and experimentally obtained temperature in the primary bay as a function of nondimensional coordinates for four beam depths (Note the axis of the figure are based on Delichatsios's formulation). The results displayed in Figure 9 indicate good agreement between the predicted and experimentally measured values. This is despite the fact that the correlation for $\mathrm{b}$ was derived from data of a single $Q^{*}$ and $1_{b} / H$ condition (see Fig. 9).

The assumptions which have been made to obtain Eq. (3) include limited entrainment and a constant heat transfer coefficient. Based on the velocity profile data shown in Figure 6 and the temperature predictions shown in Figure 9, it appears that these two assumptions are reasonably valid. Therefore, the temperature reductions in the primary bay appear to be primarily a result of heat transfer to the ceiling. A number of beam spacings and beam depths have been investigated in this

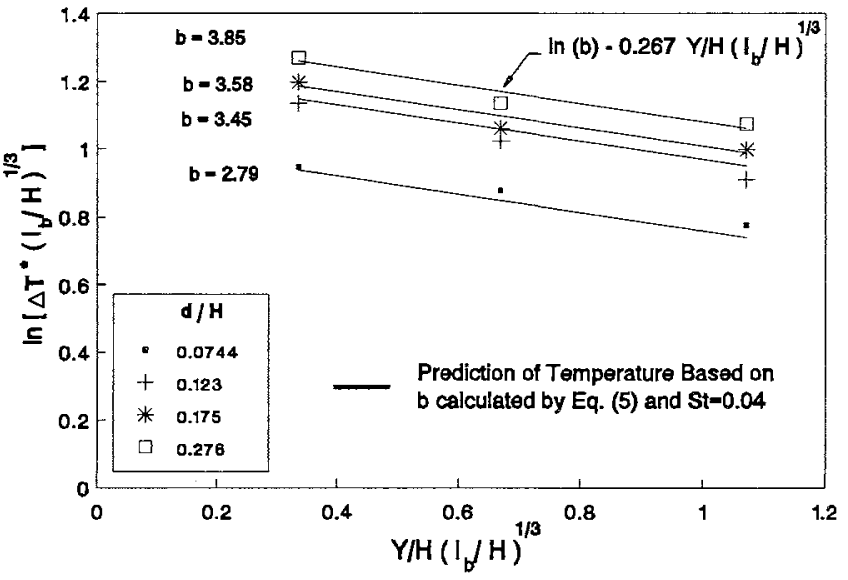
study, however, Eq. (3) may be used to predict ceiling jet temperatures in the primary bay in conjunction with the experimentally correlated pre-exponential factor and Stanton number.

\section{CONCLUSIONS}

Data collected in the primary channel indicate that the assumption of sub-critical flow and reduced entrainment is valid. It has been shown that the ceiling jet temperature as a function of distance from the fire source, within the primary bay, is a function of beam depth to height ratio and beam spacing. The ceiling jet temperature reduction is a result of heat transfer to the ceiling and beams, not as a result of air entrainment, as found in the axisymmetric ceiling jet. 
The data and results indicate that two ceiling jet flow regimes exist under a beamed ceiling. The ceiling jet temperature and velocity is most greatly affected between $0<\mathrm{d} / \mathrm{H}$ $<0.15$. Within this range, the ceiling jet has a substantial increase of the temperature within the primary bay. Even at the smallest beam depth tested $(\mathrm{d} / \mathrm{H}=0.048)$, the ceiling jet flow is significantly affected by the presence of the beams. Subsequent increases in the non-dimensional ceiling jet temperature and velocity are less dramatic for beam depth to ceiling height ratios greater than 0.2 .

The complexity of the beamed ceiling geometry makes generalization of the ceiling jet temperature and velocity data to heat detector response difficult. However, the approach presented in NFPA 72E [12] standard can be easily extended to include ceilings with obstructions using the correlations resulted from this work, within the stated limitations, along with the detector characteristics, room geometry, and the concept of a threshold fire size, $\dot{Q}_{d}$.

\section{Acknowledgement}

This work was partially funded by the student scholarship grant to Carl Koslowski from the SFPE Educational and Scientific Foundation.

\section{REFERENCES}

1. Alpert, R.L., "Fire Induced Turbulent Ceiling Jet," Tech. Rpt, No. 19722-2, Factory Mutual Res. Corp., Norwood, MA, May 1971.

2. Heskestad, G. and Delichatsios, M.A. "Environments of Fire Detectors- Phase I: Effect of Fire Size, Ceiling Height and Material," Technical Report, FMRC Serial No. 22427, Factory Mutual Research Corporation, Norwood, MA, May 1977.

3. Motevalli, V. and Marks, C.H., "Characterizing the Unconfined Ceiling Jet Under Steady State Conditions: A Reassessment," 3rd Int'l Symp. on Fire Safety Science, pp. 301-312, 1991.

4. Heskestad, G., "Model Study of ESFR Sprinkler Response Under Beamed Ceilings," Tech. Rpt., FMRC J.I. ONOE3.RU, Factory Mutual Res. Corp., Norwood, MA, 1987.

5. Bill, R.G. Jr., Kung, H-C, Brown, W.R., Hill, E.E., "Effects of Cathedral and Beamed Ceiling Construction on Residential Sprinkler Performance," Technical Report, J.I.

OM3N5.RA (3), Factory Mutual Research Corporation, Norwood, MA, February 1988.

6. Koslowski, C.C. and Motevalli, V., "Effects of Beams on Ceiling Jet Behavior and Heat Detector Operation", J. of Fire Protection Eng., Vol. 5, No. 3, pp. 97-112, 1993.

7. Delichatsios, M.A., "The Flow of Fire Gases Under a Beamed Ceiling", Combustion and Flame, 43, 1, pp. 1-10, 1981.

8. Heskestad, G., and Delichatsios, M.A., " Environments of Fire Detectors-Phase II: Effect of Ceiling Configuration, FMRC No. 22584, Factory Mutual Res. Corp., 1978.

9. Beyler, C.L., "Fire Plumes and Ceiling Jets," Fire Safety Journal, 11 (1986) 53-75.

10. Motevalli, V., Marks, C.H., and McCaffrey, B.J., "Cross-Correlation Velocimetry for Measurement of Velocity and Temperature Profiles in Low-Speed, Turbulent, NonIsothermal Flows," J. of Heat Transfer, Vol. 114, 2, p. 331-337, 1992.

11. Koslowski, C.C., "Effects of Beams and Ceiling Obstructions on Steady State Ceiling Jet Flow", M.S. Thesis, Worcester Polytechnic Institute, Worcester, MA, 1991.

12. NFPA 72-1993, National Fire Alarm Code, National Fire Protection Association, Quincy, MA, 1993. 